\title{
Mobile Video Streaming in Modern Wireless Networks
}

\author{
Mohamed Hefeeda \\ School of Computing Science \\ Simon Fraser University \\ Surrey, BC, Canada \\ mhefeeda@cs.sfu.ca
}

\author{
Cheng-Hsin Hsu \\ Deutsche Telekom R\&D Lab USA \\ 5050 El Camino Real 221 \\ Los Altos, CA 94022 \\ cheng-hsin.hsu@telekom.com
}

\begin{abstract}
Increasingly more users use mobile devices to watch videos streamed over wireless networks, and they demand more content at better quality. For example, market forecasts reveal that mobile video streaming, such as mobile TV, will catch up with gaming and music, and become the most popular application on mobile devices. In this tutorial, we will present different approaches to deliver multimedia content over various wireless networks to a large number of mobile users. We will study and analyze the main research problems in modern wireless networks that need to be addressed in order to enable efficient mobile video services. The tutorial will cover common research problems in wireless networks such as HSDPA, MBMS, WiMAX, LTE, DVB-H, MediaFLO, and ATSC M/H. After giving the preliminaries of the considered wireless network standards, we will focus on important research problems and present their solutions in details. Finally, we will discuss open problems and future research directions in mobile video. The tutorial will be composed of five parts, which are briefly described in Sec. 1-5.

Categories and Subject Descriptors: C.2.0 [Computer Systems Organization]: Computer-Communication Networks General
\end{abstract}

General Terms: Design

\section{INTRODUCTION}

We present a simple model for mobile video streaming systems, which abstracts away the common components to enable efficient mobile video streaming. We then specialize this model to several modern wireless networks, including HSDPA, MBMS, WiMAX, LTE, DVB-H, MediaFLO, and ATSC M/H. We next present the advantages/disadvantages of mobile video streaming over each of the considered wireless networks. Our comparison concentrates on their architectures, protocols, operation, deployment cost, and future potential. Our comprehensive comparison allows audience to understand the merits of individual wireless networks, and enables them to pick the wireless network that is most suitable to their needs. More importantly, our introduction clearly identifies the common components of all wireless networks. This enables general discussions on challenges and solutions in the rest of our tutorial, which can be readily applied to various wireless networks.

Copyright is held by the author/owner(s).

$M M^{\prime} 10$, October 25-29, 2010, Firenze, Italy.

ACM 978-1-60558-933-6/10/10.

\section{CHALLENGES}

First, we discuss general challenges in multimedia wireless networks. For example, mobile video streaming systems need to cope with high error rates in wireless networks, because wireless channels are vulnerable to noise, fading, shadowing, and interference. In addition, some wireless networks deploy smaller cells to enable frequency reuse for higher spectrum efficiency. In these wireless networks, mobile video streaming systems must consider mobility and handoffs for mobile users to roam among cells. Second, we present challenges on providing good video streaming Quality-of-Experience (QoE) in mobile video streaming systems, such as video quality, energy saving, and bandwidth efficiency.

\section{PROBLEMS AND RECENT RESEARCH ADVANCES}

We next discuss concrete research problems in multicast ${ }^{1}$ and unicast video streaming.

\subsection{Multicast Video Streaming}

- Prolong viewing time of mobile receivers. Multicast streaming systems transmit each video in bursts at a bit rate much higher than its encoding rate in order to save energy on mobile receivers. This is called time slicing, which requires base stations to carefully compose burst schedules to guarantee streaming quality and proper functioning of the system. Recent works, such as [2], propose burst scheduling algorithms to maximize energy saving of mobile receivers.

- Increase bandwidth efficiency of wireless networks. Higher bandwidth efficiency leads to more concurrent video streams within a given network bandwidth, and thus results in higher profits for service providers. One way to increase bandwidth efficiency is to encode videos in VBR (Variable-Bit-Rate), rather than CBR (Constant-Bit-Rate), for higher statistical multiplexing gain. Streaming VBR videos over multicast wireless networks, however, imposes more challenges to the base stations, which has been studied in a few recent works, such as [3].

- Control channel switching delay. Since multiple videos are time-multiplexed before being sent over the

\footnotetext{
${ }^{1}$ We interchangeably use multicast and broadcast in this paper.
} 
shared multicast medium, switching video channels incurs channel switching delay. Long and variable channel switching delay is annoying to users and may turn them away from the video streaming service. Recent studies $[4,8]$ propose methods to reduce the switching delay for better user experience.

- Support heterogeneous mobile receivers. Supporting mobile receivers with heterogeneous resources, such as screen resolution and decoder capability, is important for commercially-viable services. Traditional simulcast concurrently transmits multiple nonscalable streams of each video, which effectively reduces the number of videos that can be offered by the service provider. Recent works, e.g., [5], propose to multicast scalable video streams in order to support heterogeneous mobile receivers.

\subsection{Unicast Video Streaming}

- Power-aware video streaming. Power consumption of mobile receivers consists of three major components: processing (for video coding), communication (for video delivery), and background (for other parts, such as back-light). Optimally allocating the stringent energy budget between processing and communication is challenging because of the nonlinearity between transmission power and packet error rate, as well as between coding efficiency and en-/de-coding complexity. Minimizing the overall power consumption of mobile receivers is an active research topic [11].

- Rate allocation over a shared medium. Multiple video streams sharing the same wireless network compete with each other for available network bandwidth. Rate control in application layer (encoding rate) and network layer (streaming rate) must be carefully considered to avoid network congestion and late video packets. We will discuss recent developments on rate allocation such as those in [12].

- Stream adaptation for timely delivery. Since wireless networks are bandwidth constrained, video streams must be transformed into lower bit rates to ensure timely delivery. Stream adaptation can be implemented via transcoding [10] or scalable video coding [9]. Optimal stream adaptation is challenging due to the complexity of human vision systems.

- Deadline oriented packet scheduling. Mobile video systems must carefully schedule the sending times of individual video packets to guarantee ontime delivery because late packets are essentially useless. The packet scheduling problem is NP-Complete as a machine scheduling problem can be reduced to it, and a few recent works propose greedy algorithms [7] and approximation algorithms [6] to address it.

\section{OPEN RESEARCH PROBLEMS}

Several issues of mobile video streaming have not yet been rigorously studied. We will present these open problems. For example, we briefly describe an open problem of designing a hybrid streaming network in the following, while more open problems will be discussed in the tutorial.

To minimize bandwidth requirements, depending on the number of receivers for individual videos, some videos may better be multicast, while others may be more suitable to be unicast. For illustration, consider a video stream requested by a single mobile user, devoting a channel in multicast networks to it may not be bandwidth efficient. In such scenario, streaming using unicast networks is more efficient. Therefore, hybrid unicast-multicast networks lead to better bandwidth efficiency. Several works in the literature investigate the potential benefits of building such hybrid networks, e.g., [1]. However, a few design challenges in such hybrid networks remain open.

\section{CONCLUSIONS AND OUTLOOK}

We will summarize our tutorial and review the most important take-aways. Moreover, we will give service providers some guidelines on choosing the wireless networks that are suitable to them. We believe our tutorial will largely simplify the future design and implementation of mobile video streaming over modern wireless networks.

\section{REFERENCES}

[1] A. Bria. Cost-based resource management in hybrid cellular-broadcasting systems. In Proc. of IEEE Vehicular Technology Conference (VTC'05-Spring), pages 3183-3187, Stockholm, Sweden, May 2005.

[2] C. Hsu and M. Hefeeda. Broadcasting video streams encoded with arbitrary bit rates in energy-constrained mobile TV networks. IEEE/ACM Transactions on Networking, August 2009. Accepted to appear.

[3] C. Hsu and M. Hefeeda. Statistical multiplexing of variable-bit-rate videos streamed to mobile devices. $A C M$ Transactions on Multimedia Computing, Communications, and Applications, December 2009. Accepted to appear.

[4] C. Hsu and M. Hefeeda. Using simulcast and scalable video coding to efficiently control channel switching delay in mobile TV broadcast networks. ACM Transactions on Multimedia Computing, Communications, and Applications, October 2009. Accepted to appear.

[5] C. Hsu and M. Hefeeda. Flexible broadcasting of scalable video streams to heterogeneous mobile devices. IEEE Transactions on Mobile Computing, March 2010. Accepted to appear.

[6] C. Hsu and M. Hefeeda. Quality-aware segment transmission scheduling in peer-to-peer streaming systems. In Proc. of ACM Multimedia Systems (MMSys'10), pages 169-180, Scottsdale, AZ, February 2010.

[7] J. Huang, Z. Li, M. Chiang, and A. Katsaggelos. Joint source adaptation and resource allocation for multi-user wireless video streaming. IEEE Transactions on Circuits and Systems for Video Technology, 18(5):582-595, May 2008.

[8] M. Rezaei, I. Bouazizi, and M. Gabbouj. Joint video coding and statistical multiplexing for broadcasting over DVB-H channels. IEEE Transactions on Multimedia, 10(7):1455-1464, December 2008.

[9] H. Schwarz, D. Marpe, and T. Wiegand. Overview of the scalable video coding extension of the H.264/AVC standard. IEEE Transactions on Circuits and Systems for Video Technology, 17(9):1103-1120, September 2007.

[10] J. Xin, C. Lin, and M. Sun. Digital video transcoding. Proceedings of the IEEE, 93(1):84-97, January 2005.

[11] J. Zhang, D. Wu, S. Ci, H. Wang, and A. Katsaggelos. Power-aware mobile multimedia: a survey. Journal of Communications, 4(9):600-613, October 2009.

[12] X. Zhu and B. Girod. Video streaming over wireless networks. In Proc. of European Signal Processing Conference (EUSIPCO'07), pages 1462-1466, Poznan, Poland, September 2007. 